\title{
Philosophiques
}

\section{Gilles Lane, À qui bon la philosophie? Longueuil, Le Préambule, 1982, 118 p.}

\section{René Pellerin}

Volume 11, numéro 1, avril 1984

URI : https://id.erudit.org/iderudit/203254ar

DOI : https://doi.org/10.7202/203254ar

Aller au sommaire du numéro

Éditeur(s)

Société de philosophie du Québec

ISSN

0316-2923 (imprimé)

1492-1391 (numérique)

Découvrir la revue

Citer ce compte rendu

Pellerin, R. (1984). Compte rendu de [Gilles Lane, À qui bon la philosophie?

Longueuil, Le Préambule, 1982, 118 p.] Philosophiques, 11(1), 213-216.

https://doi.org/10.7202/203254ar d'utilisation que vous pouvez consulter en ligne.

https://apropos.erudit.org/fr/usagers/politique-dutilisation/ 
Gilles LANE, À quoi bon la philosophie? Longueuil, Le Préambule, 1982, $118 \mathrm{p}$.

\section{par René Pellerin}

Le dernier article de Lane date de 1979 ( « Il est impossible d'intervenir dans le cours des événements " in Dialogue, XVIII, 1, 1979 : 1-13) et son dernier livre de 1973 (L'urgence du présent, essai sur la contre-culture, Montréal, P.U.Q.). Ce que Lane présente cette année à ses lecteurs est marqué par la sobriété et la radicalité. Après la philosophie du langage, la philosophie des sciences et ce qu'il conviendrait d'appeler avec beaucoup de nuances une " ontologie » (parce que Lane ne craint pas de s'intéresser à la " réalité " et à ce qu'il nomme prudemment " le réel "), il s'attaque à un problème de taille. En posant en effet, de façon assez anachronique, la question de l'importance de l'investissement philosophique, question que l'on verrait peutêtre mieux avant ce genre de démarche qu'après, Lane tente de comprendre l'enjeu du savoir.

Contrairement à beaucoup de productions dites assez vaguement « philosophiques » l'auteur se tient ici dans la ligne des préoccupations des grands philosophes de l'histoire. L'essentiel du livre tourne autour de la recherche de la finalité de l'activité même de chercher les finalités, les raisons, les causes et les « essences ». Qu'est-ce que le philosophe veut au juste obtenir ou vivre par la philosophie et dans la philosophie ? Quel est au juste l'enjeu qui semble tenir tant à cœur à ceux qui investissent dans la démarche cognitive ? On trouvera ici une hypothèse (présentée comme telle par l'auteur) qui s'inscrit dans la ligne de ce que Lane propose depuis ses premiers livres (Étre et Langage, Paris, Aubier, 1970), à savoir que la réalité que l'on ne veut pas « manquer » est à concevoir sur le modèle de la « rencontre " et non sur celui de l'appropriation abstraite.

Il ne s'agit donc ni d'une chose, ni d'une idée mais d'une réalité capable de « rejoindre " la conscience qui " l'attend ". C'est dans ce langage métaphorique que Lane cherche à comprendre les possibilités de sens des objectifs plus affirmés que justifiés, et plus critiquables que l'ont prétendu ceux qui s'y sont consacrés sans en voir les implications, disons, " existentielles " et personnelles.

Ce livre décevra ceux qui se préoccupent de la philosophie dans sa forme et ses objectifs institutionnels. Il décevra aussi ceux qui en attendent des arguments à offrir aux instances administratives, pour justifier la rentabilité socio-culturelle de la philosophie. Pour l'auteur, la philosophie n'est rien de moins, et rien de plus, qu'une sorte de "vocation " dont celui qui l'assume attend une "valorisation personnelle " (ce qui n'empêche pas, évidemment, qu'on veuille l'obtenir par des engagements politiques ou culturels dont d'autres pourront bénéficier). Lane est tout particulièrement sensible au caractère disons « intime » de l'expérience de la compréhension (en ce sens il est très cartésien). 
L'analyse que l'auteur mène, à travers Aristote et Wittgenstein d'abord, et pour son propre compte ensuite, tombe cependant à point dans une situation où l'on découvre un certain caractère aliénant dans la valorisation des choses au détriment des hommes, dans la valorisation des structures au détriment des expériences, dans la valorisation des concepts au détriment des « vécus ». Lane est à ce sujet très nettement opposé aux questions qui font oublier le sujet questionnant et les enjeux des réponses. Avec un courage qu'il faut lui reconnaître et une attention aux événements " microscopiques " de l'existence, attention qui permet au lecteur de se reconnaître dans sa quotidienneté et de vérifier dans sa propre expérience ce que Lane veut « pointer " comme il le dit lui-même, il s'attaque à un problème que les nonphilosophes et les " victimes " de la philosophie institutionnelle adressent fréquemment mais vainement au philosophe qui semble, lui, tenir à ne pas parler de " ces choses-là ".

À travers les convictions, les espoirs implicites, la ténacité et les exhortations plus ou moins déguisées des philosophes, l'auteur cherche ce qu'on pourrait trouver d'important à philosopher, en philosophie et par la philosophie. Après avoir établi qu'il s'agit d'éviter les illusions et que cet évitement vise une certaine libération, l'auteur se demande, assez curieusement, ce que feraient les philosophes une fois en possession de cette liberté et de cette connaissance " authentique ». Accéder à la réalité plutôt qu'à ses apparences ou accéder à ce qui est réel plutôt que de se laisser obnubiler par des illusions (ne serait-ce que celle qu'il y a de la "présence " ou de la " substance ", ne semble pas pour Lane la fin de l'histoire du philosophe. Il se demande en effet ce qu'il y a d'intéressant dans la connaissance authentique, compte tenu du fait qu'il semble que ce ne soit pas l'objet connu qui importe mais son appropriation cognitive.

On a l'impression que l'auteur veut nous mener en un lieu particulier et que les raisons insatisfaisantes apportées par les philosophes pour justifier leur entreprise ne sont finalement insatisfaisantes que parce que l'auteur n'y trouve pas la sienne. Mais n'est-ce pas là l'inévitable effet d'un travail qui ne se développe pas au fil de l'inspiration, auquel cas on ne craindrait pas qu'il nous mène là où l'auteur veut nous mener puisque, justement, l'auteur ne saurait pas (ou pas encore) lui-même où il va. Et Lane semble bien savoir où il va.

Or, et c'est en partie ce que ce livre a d'étonnant, l'auteur, qui veut que nous comprenions quelque chose, en acceptant l'hypothèse qu'il nous présente, nous fait vivre précisément ce qu'il pense que les philosophes vivent lorsqu'ils cherchent à « saisir » le réel dans « l'événement " qu'est la connaissance authentique. Le lecteur cherche comme une pensée qui serait attendue de lui par l'auteur et cette pensée, c'est que le philosophe « se comporte (. . .) comme s'il cherchait à découvrir ce qui ne saurait être qu'une pensée attendue de lui personnellement " (p. 91). Cette étrange implication du lecteur lui-même dans le problème qui lui est posé crée une sensation de complicité assez fascinante. Le vertige de la régression à l'infini des justifications de la 
justification s'arrête alors dans une expérience strictement personnelle qui constitue pour Lane «l'équivalent » d'une « rencontre » avec du « nonmoi » selon l'expression empruntée à Russell, non-moi qui serait une altérité capable de "viser " la conscience du philosophe et « d'attendre » quelque chose de lui, personnellement. L'expérience de la connaissance authentique constituerait la réussite de cette visée.

L'auteur répond de façon assez désinvolte à l'objection de l'anthropomorphisme en laissant planer le soupçon que le refus de reconnaître la possibilité à une autre réalité qu'humaine de pouvoir "viser " ou " atteindre " n'est pas moins anthropomorphique en ce qu'il implique la prétention que seuls les humains peuvent faire certaines choses.

Le philosophe, comme n'importe qui cherchant une connaissance authentique, trouverait dans l'événement de la connaissance authentique une "valorisation de lui-même » et sortirait de l'insignifiance et de l'absurdité en vivant l'expérience d'être " atteint » personnellement. (p. 97) Lane précise que cette motivation n'est pas constante et universelle mais qu'elle permet de comprendre le comportement de nombreux philosophes à certains moments de leur recherche et de leur expérience.

La fonction que prend la philosophie dans ce contexte est éminemment critique :

" le rôle social du philosophe consisterait moins à transmettre des connaissances particulières sur tel ou tel objet (c'est-à-dire d'instaurer dogmatiquement des visions arrêtées du monde) qu'à inviter les autres à se distancier momentanément de leurs visions habituelles et accaparantes, pour attendre à leur tour activement, et "à travers " ces visions, l'occurrence du type d'événement dont eux aussi auraient personnellement besoin qu'il se produise, à certains moments. »P. 110

Malgré l'indéniable valeur de cette hypothèse, il faut cependant admettre quelques lacunes et quelques insuffisances. On comprend mal en effet le souci des philosophes de se convaincre les uns les autres, ou de se réfuter les uns les autres s'il s'agit finalement de vivre une expérience personnelle de compréhension " authentique ". Serait-ce que nous serions si préoccupés des autres que nous voudrions qu'ils vivent les mêmes expériences et parviennent à la même compréhension dont l'authenticité serait en quelque sorte garantie par son universalité ?

Lane néglige par ailleurs assez radicalement le philosophe qui a le plus violemment critiqué les aspirations contemplatives et l'individualisme des philosophes qui l'avaient précédé, à savoir Karl Marx. Il est étonnant qu'il ne confronte pas la dimension dangereusement "solipsiste " de son hypothèse à ce courant de contestation de la philosophie. Notons également que la façon dont les termes " authenticité » et " personnalisation » sont utilisées est parfois ambiguë et que l'expérience de l'évidence subjective ne semble pas souvent avoir été dans l'histoire reconnue comme suffisante pour parler d'authenticité. La connaissance « personnelle » pour sa part est largement 
dépendante d'une conception fort imprécise de ce qu'est « la personne » et n'a finalement de sens que dans les marges de l'idéologie humaniste, ce qui, bien que n'étant pas un tort, limite passablement l'univocité de l'interprétation.

En bref ce livre est stimulant et réussit fort bien à faire ce que l'auteur propose précisément comme objectif de la pratique sociale de la philosophie, c'est-à-dire " pointer » vers ce qui pourrait être le terme et l'attente de la philosophie et du philosophe. Il est à souhaiter que le langage clair et la rigueur de l'argumentation constitueront des indices de son accessibilité à tous ceux qui s'interrogent à bon droit sur les enjeux de ce qui se présente comme un' sommet de l'activité cognitive. L'originalité de la pensée et la rafraîchissante infidélité à la "mode philosophique " au profit d'une radicalité peu fréquente chez les partisans de la philosophie à résultats automatiques (que Lane caricature d'ailleurs un peu méchamment en les renvoyant. à la " phénoménologie du baiser ou à l'analyse structurale du hockey »!) n'est pas sans faire penser que la production québécoise en philosophie est peut-être plus importante et intéressante dans les milieux académiques que dans le monde des " hommes réels " comme disait Marx.

Département de philosophie

Collège Laflèche, Trois-Rivières 